http://jmscr.igmpublication.org/home/ ISSN (e)-2347-176x ISSN (p) 2455-0450

crossref DOI: https://dx.doi.org/10.18535/jmscr/v7i10.83

\title{
Prevalence of Thyroid Disorders in Chronic Kidney Disease Patients in North Indian Population
}

\author{
Authors \\ Dr Arun Kumar ${ }^{1}$, Dr Sumukh ${ }^{2}$ \\ ${ }^{1}$ Medical Officer District Hospital, Jhanshi \\ ${ }^{2}$ Medical Officer, Agra, UP, India
}

\begin{abstract}
The thyroid produces hormones (T3 and T4) that have many actions including metabolism, development, protein synthesis, and the regulation of many other important hormones. Any dysfunction in the thyroid can affect the production of thyroid hormones (T3 and T4) which can be linked to various pathologies throughout the body.

Method: A prospective cross-sectional study was conducted. All the 100 cases included in the study were classified into stages of CKD according to the glomerular filtration rate (GFR) calculated by CockcroftGault formula. Staging of CKD was done according to the KDIGO guidelines.

Result: Among all the 100 cases, patients of Stage II were 10\%, 16\% patients were Stage III, 22\% patients were Stage IV, and 52\% of patients were Stage V. There were 33 CKD patients with SCH, 3 patients (9.09\%) were Stage II CKD, 1 patient (3.03\%) had Stage IIIA, 5 patients (15.15\%) had Stage IIIB, 9 patients (27.27\%) had Stage IV, and 15 patients (45.45\%) had Stage V CKD. There were 20 CKD patients with overt hypothyroidism, of which 3 patients (5\%) had Stage II CKD, 1 patient (5\%) had Stage IIIA, 2 patients (10\%) had Stage IIIB, 8 patients (40\%) had Stage IV, and 8 patients (40\%) had CKD Stage $V$.

Conclusion: Among them, 53\% of patients had thyroid disorder. The most common thyroid hormone disorder was SCH (33\%) followed by clinical hypothyroidism (20\%), which was higher than that has been reported previously. The severity of thyroid dysfunction increases with progressive fall in GFR.
\end{abstract}

\section{Introduction}

The thyroid produces hormones (T3 and T4) that have many actions including metabolism, development, protein synthesis, and the regulation of many other important hormones. Any dysfunction in the thyroid can affect the production of thyroid hormones (T3 and T4) which can be linked to various pathologies throughout the body. One of the most important conditions that have been less studied is thyroid hormone levels and how they affect the progression of CKD. Disorders in renal function have been seen to coexist with specific levels of thyroid hormone. The 2010 Global Burden of Disease study mentions chronic kidney disease (CKD) as the 27th cause of mortality in 1990, rising to 18th in 2010, second only to that for HIV, AIDS. ${ }^{[3]}$ Kidneys regulate the metabolism, degradation, and excretion of thyroid hormones; impaired kidney functions alter hormone production, distribution, and excretion. Data suggest that predialysis patients with CKD have 
an increased risk of hypothyroidism, with many cases being subclinical ${ }^{[1,2]}$

Primary hyperthyroidism is rare, while the prevalence of subclinical hypothyroidism ( $\mathrm{SCH})$ and overt hypothyroidism is increased in patients with CKD. ${ }^{[4-7]}$ Hypothyroidism shares common symptoms with CKD such as pallor, hypothermia, and asthenia, with diagnosis resting largely on biochemical tests. $^{[8]}$

\section{Aims and Objectives}

This study was conducted to study the prevalence of thyroid dusfunction in patients with chronic kidney disease and to find the correlation between thyroid dysfunction and severity of of renal diseases.

\section{Methods}

A prospective cross-sectional study was conducted in the Post Graduate Department of Medicine, S. N. Medical College, Agra, Uttar Pradesh, India, between june 2018 and June 2019. A total of 100 patients were included in this study after fulfilling the inclusion criteria. We included CKD patients aged $>20$ years. All the 100 cases included in the study were classified into stages of CKD according to the glomerular filtration rate (GFR) calculated by Cockcroft-Gault formula. Staging of CKD was done according to the KDIGO guidelines. ${ }^{[10]}$

Patients undergoing peritoneal dialysis or hemodialysis, acute illness of any nature, recent surgery, trauma, those with diabetes mellitus and liver diseases; those on drugs altering thyroid profile such as amiodarone, steroids, dopamine, phenytoin, beta- blocker, estrogen pills, iodine- containing drugs; and patients with any previous long- term history of thyroid disorders were excluded from the study. An informed written consent was taken from all the cases before enrolling into the study and a detailed history taking and general examination were done. Blood samples were collected from the participants and analyzed for free triiodothyronine (FT3), free thyroxine (FT4), and thyroid stimulating hormone (TSH) by electrochemiluminescence technique using the kits by Advia Centaur XP analyzer system of Siemens (siiemens healthineers limited, Siemens healthcare GmbH, Henkestr, 127, 91052 Erlangen, Germany).

\section{Results}

In our study 100 patients of CKD who were on conservative management fulfilling the criteria for CKD were studied, among these 60 were males and 40 were females, their age varied from 20-70 years.

Table 1 Distribution of cases on the basis of creatinine clearance in chronic kidney patients who were on conservative management.

\begin{tabular}{|l|c|c|}
\hline Stages of CKD & $\begin{array}{c}\text { Creatinine clearance } \\
\left(\mathrm{ml} / \mathrm{min} / 1.73 \mathrm{~m}^{2}\right)\end{array}$ & Cases \\
\hline I & $>90$ & 0 \\
\hline II & $60-89$ & 10 \\
\hline IIIa & $45-59$ & 05 \\
\hline IIIb & $30-44$ & 11 \\
\hline IV & $15-29$ & 22 \\
\hline V & $<15$ & 52 \\
\hline TOTAL & & 100 \\
\hline
\end{tabular}

Among all the 100 cases, patients of Stage II were $10 \%, 16 \%$ patients were Stage III, $22 \%$ patients were Stage IV, and $52 \%$ of patients were Stage V. Among the patients studied, most belonged to Stage V.

Table-2 Distribution of thyroid stimulating hormone values in thyroid disorders cases

\begin{tabular}{|l|c|c|c|c|c|}
\hline & $\mathrm{N}$ & Mean & SD & t-value & p-value \\
\hline SCH & 33 & 8.44 & 0.79 & -15.5694 & $<0.0001$ \\
\hline Clinical Hypo & 20 & 13.29 & 1.25 & & \\
\hline
\end{tabular}

In the table 2 there were $33 \mathrm{CKD}$ patients with subclinical hypothyroidism with mean TSH value of 8.44 and 20 CKD patients with clinical hypothyroidism with mean TSH value of 13.29. 
Table-3 Distribution of free thyroxin values in thyroid disorders cases

\begin{tabular}{|l|c|c|c|c|c|}
\hline & $\mathrm{N}$ & Mean & SD & t-value & p-value \\
\hline SCH & 33 & 11.89 & 1.21 & 15.009 & $<0.0001$ \\
\hline Clinical HYPO & 20 & 7.23 & 1.02 & & \\
\hline
\end{tabular}

In the table there were $33 \mathrm{CKD}$ patients with subclinical hypothyroidism with mean FT4 value of 11.89 and 20 CKD patients with hypothyroidism with mean FT4 value of 7.23.

Table 4 Prevalence of subclinical hypothyroidism and clinical hypothyroidism in different stages of CKD.

\begin{tabular}{|l|c|c|c|c|c|c|}
\hline Stages of CKD & $\begin{array}{c}\text { SCH } \\
(\mathrm{n}=33)\end{array}$ & & $\begin{array}{c}\text { Clinical Hypo } \\
(\mathrm{n}=20)\end{array}$ & & $\begin{array}{c}\text { Total } \\
(\mathrm{n}=53)\end{array}$ & \\
\hline & No & $\%$ & No. & $\%$ & No. & $\%$ \\
\hline II & 3 & 9.09 & 1 & 5.0 & 4 & 7.55 \\
\hline IIIa & 1 & 3.03 & 1 & 5.0 & 2 & 3.77 \\
\hline IIIb & 5 & 15.15 & 2 & 10 & 7 & 13.21 \\
\hline IV & 9 & 27.27 & 8 & 40 & 17 & 32.08 \\
\hline V & 15 & 45.45 & 8 & 40 & 23 & 43.40 \\
\hline Total & 33 & 100 & 20 & 100 & 53 & 100 \\
\hline
\end{tabular}

In this table Cases of clinical and subclinical hypothyroidism categorized according to glomerular filtration rate and stage of chronic kidney disease. There were 33 CKD patients with $\mathrm{SCH}, 3$ patients $(9.09 \%)$ were Stage II CKD, 1 patient $(3.03 \%)$ had Stage IIIA, 5 patients $(15.15 \%)$ had Stage IIIB, 9 patients $(27.27 \%)$ had Stage IV, and 15 patients $(45.45 \%)$ had Stage V CKD. There were $20 \mathrm{CKD}$ patients with overt hypothyroidism, of which 3 patients $(5 \%)$ had Stage II CKD, 1 patient (5\%) had Stage IIIA, 2 patients (10\%) had Stage IIIB, 8 patients (40\%) had Stage IV, and 8 patients $(40 \%)$ had CKD Stage V.

\section{Discussion}

Patients with CKD often have signs and symptoms suggestive of thyroid dysfunction and hence the diagnosis of thyroid disease in these patients has obvious prognostic implications. Thyroid autoimmunity and subclinical primary hypothyroidism are highly prevalent in CKD patients not requiring long- term dialysis treatment ${ }^{[9]}$. The present study was aimed to assess the prevalence of thyroid dysfunction in CKD and to determine the correlation between thyroid dysfunction and severity of renal disease.

JOAN C. LO et al in their study show that among 14623 adults participants with serum creatinine and thyroid function test results, the mean age was 48.7 and $52.6 \%$ were women. The prevalence of hypothyroidism increased with lower levels of GFR occurring in $5.4 \%$ of subjects with GFR $>90$, $10.9 \%$ with GFR 60-89, 20.4\% with GFR 45$59,23 \%$ with GFR $30-44$, and $23.1 \%$ with GFR $<30 \quad$ ( $<<0.001$ for trend). Overall $56 \%$ of hypothyroidism cases were considered subclinical. Compared with GFR $>90 \mathrm{ml} / \mathrm{min} / 1.73 \mathrm{~m}^{2}$, reduced GFR was associated with an increased risk of hypothyroidism.

In a study by Chandra et al $\mathrm{SCH}$ and clinically apparent hypothyroidism have been reported to occur in $\sim 40 \%$ and $16 \%$ of patients, respectively, with CKD not requiring renal replacement therapy. ${ }^{[12]}$

This study differs from these previous observations by demonstrating a prevalence of SCH and clinical hypothyroidism (53\%). An increased prevalence of subclinical and clinical primary hypothyroidism in persons with reduced estimated GFR (eGFR) independent of age and gender was seen in this study. This is in line with the observation made by Chonchol et al. ${ }^{[11]}$ Our study included 100 cases of CKD, out of which 53 cases were having thyroid dysfunction in which 20 patients had clinical hypothyroidism and 33 patients had $\mathrm{SCH}$. In this study, the absolute prevalence of hypothyroidism in the lower GFRs 
was higher than that reported in other studies, which may be due to the smaller sample size in the present study. Majority of the patients in this study fell in the CKD Stage IV/V category, which could be due to the fact that most of the CKD patients referred to this tertiary care center had a low GFR.

\section{Conclusions}

In our study, population of $100 \mathrm{CKD}$ patients who were on conservative management were studied. Among them, 53\% of patients had thyroid disorder. The most common thyroid hormone disorder was $\mathrm{SCH}$ (33\%) followed by clinical hypothyroidism (20\%), which was higher than that has been reported previously. The severity of thyroid dysfunction increases with progressive fall in GFR.

The role of clinical or SCH on physical function, cognitive function, quality of life, and depression in CKD is unknown. However, health professionals caring for patients with CKD should be cognizant that CKD and hypothyroidism may exhibit overlapping symptom complexes. Hyperthyroidism which was found in some previous studies was not found in this study. Subclinical primary hypothyroidism is more common in persons with CKD not requiring chronic dialysis compared with those with normal kidney function in a large sample of unselected outpatient adults

\section{References}

1. Castellano M, Turconi A, Chaler E, Maceiras M, Rivarola MA, Belgorosky A. Thyroid function and serum thyroid binding proteins in prepubertal and pubertal children with chronic renal insufficiency receiving conservative treatment, undergoing hemodialysis, or receiving care after renal transplantation. J Pediatr 1996;128:784- 90.

2. Ramírez G, Jubiz W, Gutch CF, Bloomer HA, Siegler R, Kolff WJ, et al. Thyroid abnormalities in renal failure. A study of
53 patients on chronic hemodialysis. Ann Intern Med 1973;79:500- 4.

3. Jha V, Garcia- Garcia G, Iseki K, Li Z, Naicker S, Plattner B, et al. Chronic kidney disease: Global dimension and perspectives. Lancet 2013;382:260- 72.

4. Schaefer F, Anderzej W, Eberhard R. The patient with failing renal function, endocrine disorders. In: Alex DM, Stewart CJ, Eberhard R, David N, Krr S, Jean- Pierre G, editors. Oxford Textbook of Clinical Nephrology. New York, USA: Oxford University Press; 1997. p. 3187- 94.

5. Takeda S, Michigishi T, Takazakura E. Iodine- induced hypothyroidism in patients on regular dialysis treatment. Nephron 1993;65:51- 5.

6. Kaptein EM, Quion- Verde H, Chooljian CJ, Tang WW, Friedman PE, Rodriquez $\mathrm{HJ}$, et al. The thyroid in end- stage renal disease. Medicine (Baltimore) 1988;67:187- 97.

7. Gomez- Pan A, Alvarez- ude F, Yeo PB, Hall R, Evered DC, Kerr DN. Function of the hypothalamic- hypophyseal- thyroid axis in chronic renal failure. Clin Endocrinol 1996;2:567- 74.

8. Mohamedali M, Reddy Maddika S, Vyas A, Iyer V, Cheriyath P. Thyroid disorders and chronic kidney disease. Int $\mathbf{J}$ Nephrol 2014;2014:520281.

9. Targher G, Chonchol M, Zoppini G, Salvagno G, Pichiri I, Franchini M, et al. Prevalence of thyroid autoimmunity and subclinical hypothyroidism in persons with chronic kidney disease not requiring chronic dialysis. ClinChem Lab Med 2009;47:1367- 71.

10. Stevens PE, Levin A; Kidney Disease: Improving Global Outcomes Chronic Kidney Disease Guideline Development Work Group Members. Evaluation and management of chronic kidney disease: Synopsis of the kidney disease: Improving 
global outcomes 2012 clinical practice guideline. Ann Intern Med 2013;158: 825- 30.

11. Chonchol M, Lippi G, Salvagno G, Zoppini G, Muggeo M, Targher G, et al. Prevalence of subclinical hypothyroidism in patients with chronic kidney disease. Clin J Am SocNephrol 2008;3:1296- 300.

12. Chandra A. Prevalence of hypothyroidism in patients with chronic kidney disease: A cross-sectional study from North India. Kidney Res Clin Pract 2016;35:165- 8. 